\title{
Early-Onset Posterior Capsule Opacification: Incidence, Severity, and Risk Factors
}

\author{
Xiaoxun $\mathrm{Gu} \cdot$ Xiaoyun Chen · Guangming Jin · Lanhua Wang • \\ Enen Zhang · Wei Wang · Zhenzhen Liu (D) · Lixia Luo
}

Received: August 9, 2021 / Accepted: September 14, 2021 / Published online: November 2, 2021

(C) The Author(s) 2021

\begin{abstract}
Introduction: To evaluate the incidence, severity, and risk factors of early-onset posterior capsule opacification (PCO) following uneventful phacoemulsification and intraocular lens (IOL) implantation.

Methods: Patients with cataracts who underwent phacoemulsification and IOL implantation surgery for 3 months from September 2019 to April 2020 were enrolled. All the subjects completed a comprehensive ocular examination. Retroillumination images of the posterior capsule were obtained using a slit lamp with imaging system, and PCO was graded by two ophthalmologists. Univariate and multivariate logistic regression analyses were performed to assess the risk factors for PCO.

Results: A total of 1039 subjects were enrolled, with mean age $66.68 \pm 11.43$ years and $42.06 \%$ were male. The incidence of early-onset PCO in
\end{abstract}

Xiaoxun $\mathrm{Gu}$ and Xiaoyun Chen contributed equally to this work.

X. Gu $\cdot$ X. Chen · G. Jin · L. Wang - E. Zhang .

W. Wang · Z. Liu $(\square) \cdot$ L. Luo $(\square)$

State Key Laboratory of Ophthalmology, Zhongshan

Ophthalmic Center, Sun Yat-Sen University,

Guangzhou, Guangdong, China

e-mail: liuzhenzhen@gzzoc.com

L. Luo

e-mail: luolixia@gzzoc.com the 3 months after cataract surgery was $29.93 \%$, and PCO of grade 3 and grade 4 was present in 31 patients (2.98\%). Patients with complicated cataract had a higher incidence of PCO than age-related cataract, especially for patients with previous pars plana vitrectomy (PPV) surgery $(P<0.001)$. Moreover, the incidence of PCO increased with the deficiency of capsulorhexis-IOL overlap $(P<0.001)$. Multivariate logistic regression also showed that previous PPV surgery (OR 2.664, $P=0.003$ ) and incomplete capsulorhexis-IOL overlap were risk factors for PCO (180-360 overlap: OR 2.058, $P<0.001 ;<180^{\circ}$ overlap: OR 5.403, $\left.P<0.001\right)$. Conclusions: Larger capsulorhexis and PPV surgery history contribute to the occurrence of early-onset $\mathrm{PCO}$, indicating that primary posterior continuous curvilinear capsulorhexis can be considered during cataract surgery for patients with PPV history.

Keywords: Capsulorhexis; Cataract surgery; Early-onset posterior capsule opacification; Intraocular lens; Risk factors 


\section{Key Summary Points}

Why carry out this study?

Early-onset posterior capsule opacification (PCO) can dramatically reduce patients' postoperative satisfaction and its subsequent treatment leads to a large financial burden on the health care system.

However, the incidence of early-onset PCO and its risk factors are still unknown.

What was learned from the study?

PCO can occur in a short time after cataract surgery. The incidence of earlyonset PCO was 29.93\%, including 2.98\% of patients with grade $3+$ PCO.

Previous pars plana vitrectomy (PPV) surgery and incomplete capsulorhexis-intraocular lens (IOL) overlap are the risk factors for early-onset PCO.

Primary posterior continuous curvilinear capsulorhexis can be performed during cataract surgery for patients with PPV history.

\section{INTRODUCTION}

Posterior capsule opacification (PCO) is the most common postoperative complication with the prevalence about $50 \%$ in the 5 years after cataract surgery [1]. However, if PCO occurs early after surgery it dramatically reduces patient satisfaction because of the increase of light scattering and the reduction of visual performance [2, 3]. Neodymium-yttrium aluminum garnet (Nd:YAG) capsulotomy is commonly used for treatment for PCO. Nevertheless, it may induce vision-related complications such as ocular inflammation, cystoid macular edema, and retinal detachment [4-6], and gives rise to a large financial burden on the health care system $[7,8]$.
Previous studies have reported that primary posterior continuous curvilinear capsulorhexis (PCCC) during cataract surgery is a valid and safe way to prevent PCO [9-11]. It not only significantly reduces PCO formation and the need for Nd:YAG laser capsulotomy but also has a low rate of complications such as retinal detachment and endophthalmitis [9-11]. Although surgery age, intraocular lens (IOL) material and design, and surgical technique are associated with long-term PCO, the incidence of early-onset PCO and its risk factor are still unknown [1, 12-14]. Therefore, figuring out the risk factors for early-onset PCO and performing PCCC combined with phacoemulsification for such patients may help to reduce the need of $\mathrm{Nd}$ :YAG treatment in the early postoperative period and improve patient satisfaction.

In this cross-sectional study, we focused on early-onset PCO 3 months after cataract surgery, analyzed its characteristics, and identified the main risk factors for it in a large sample.

\section{METHODS}

\section{Study Population}

This cross-sectional study was conducted at Zhongshan Ophthalmic Center, Sun Yat-sen University, Guangzhou, China. It was approved by the Zhongshan Ophthalmic Center Institutional Review Board (No. 2019 KYPJ033) and was conducted in accordance with the Declaration of Helsinki. Written informed consent was obtained from each participant. Patients were not offered any compensation or incentives to join this study. A total of 1039 patients (1039 eyes) that underwent phacoemulsification and IOL implantation from September 2019 to April 2020 and participated in 3-month follow-up were enrolled. Subjects with the following conditions were excluded: (1) age less than 18 years; (2) ocular diseases affecting anterior structures such as lens subluxation, ocular trauma, corneal scar, and uveitis; (3) a history of retinal vascular disorders such as central retinal vein occlusion (CRVO), branch retinal vein occlusion (BRVO); (4) poor pupil mydriasis (less than $6 \mathrm{~mm}$ ) and low image 
quality affecting capsulorhexis and PCO evaluation; (5) failure to finish all the examinations leading to incomplete data. The procedure of how the participants enrolled in this study is shown in Fig. 1.

\section{Study Procedures}

All the patients fulfilled a structured questionnaire that contained demographic data (age, gender), medical history (ophthalmic and systemic history), and lifestyle (smoking, drinking, and body mass index) preoperatively. Ocular examinations performed before surgery and the surgical procedures were reported in Chen's previous study [15]. Briefly, lens thickness (LT), axial length (AL), and anterior chamber depth (ACD) were measured by IOL Master 700 (Carl Zeiss, Meditec, Dublin, CA, USA) before the surgery. Afterwards, all the patients received standard phacoemulsification for cataract extraction and an in-the-bag folded IOL implantation surgery by experienced cataract surgeons in 1 week. Electronic medical records were checked retrospectively and IOL types were recorded.

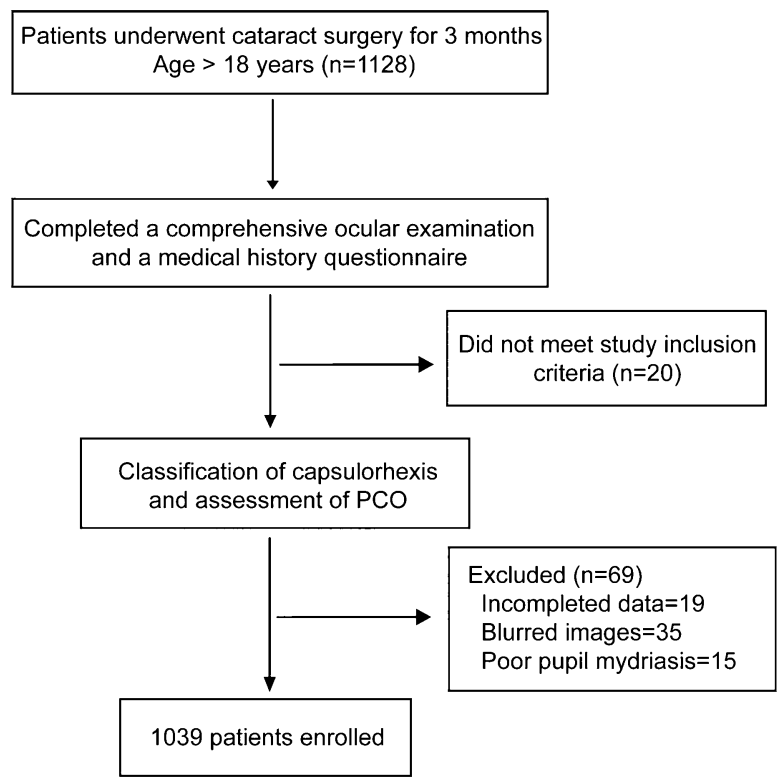

Fig. 1 Flowchart of recruitment of participants
All the eligible participants were followed up at 3 months after cataract surgery. After completing the general ophthalmic examinations (visual acuity, intraocular pressure, and slit lamp biomicroscopy), all the patients accepted a mixture of $0.5 \%$ tropicamide and $0.5 \%$ phenylephrine hydrochloride eye drops (Santen, Suzhou, China) every 5 min three times for mydriasis. Retroillumination images of the posterior capsule were collected with a HaagStreit BQ-900 slit lamp with EyeCap imaging system (Haag-Streit International, Koeniz, Switzerland). Fundus examination was conducted with Kowa nonmyd WX 3D camera (2D/ 3D non-mydriatic retinal camera, Kowa, Japan) after pupil dilation.

\section{Posterior Capsule Opacification Grading and Classification of Capsulorhexis-Intraocular Lens Overlap}

Retroillumination images of the posterior capsules obtained from all patients were graded by two ophthalmologists (XX-G and XY-C) according to Congdon's study: grade 0 (without PCO), no opacity or opacity appeared only on peripheral capsule; grade 1 , wrinkling or opacity of the capsule limited in a circle $4 \mathrm{~mm}$ in diameter and centered on the visual axis, but the posterior polar retina could be viewed clearly; grade 2, central/paracentral opacity worse than grade 1 that affected the detailed observation of macula slightly, but had no effects on the observation of cup/disc ratio; grade 3 , central/paracentral opacity worse than grade 2 , and making the cup/disc ratio difficult to ascertain; grade 4 , central/paracentral opacity as defined above, but making fundus observation difficult or impossible [16]. According to Congdon's study, we defined PCO grade 3 or above as PCO with visual impact [16]. Controversial grading images were reassessed by a senior ophthalmologist. Representative images of different PCO grades are shown in Fig. 2.

The classification of capsulorhexis-IOL overlap was evaluated as follow: $360^{\circ}$ overlap, capsulorhexis overlapping IOL edge continuously for four quadrants; $180-360^{\circ}$ overlap, 


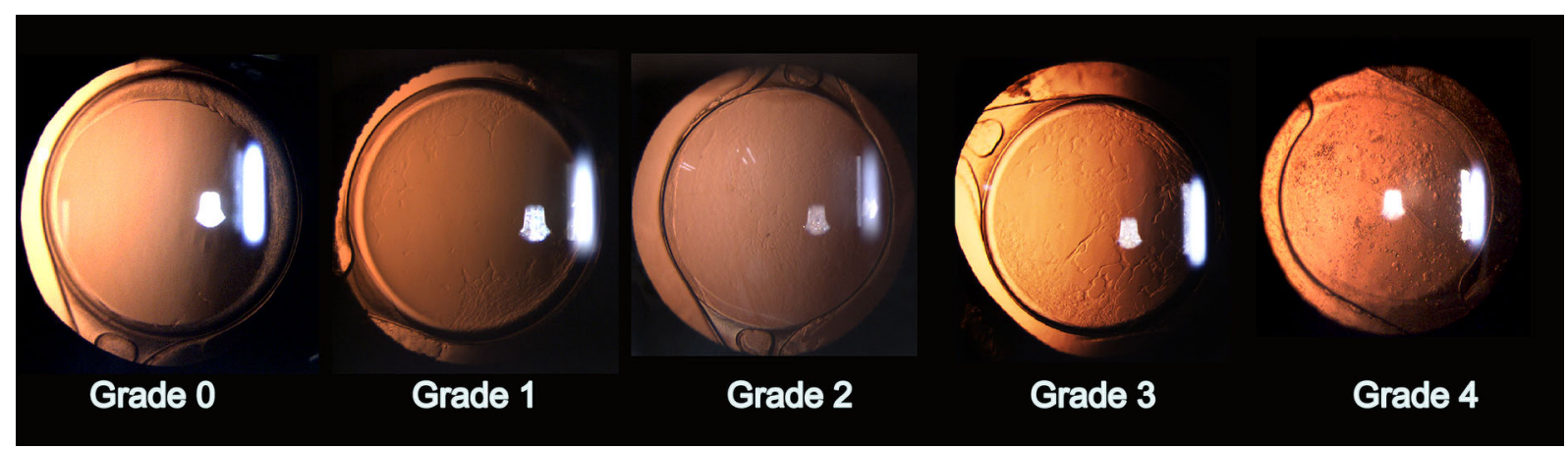

Fig. 2 Representative images of different grades of posterior capsule opacification

capsulorhexis overlapping IOL edge at least two quadrants but fewer than four quadrants; $<180^{\circ}$ overlap, capsulorhexis overlapping IOL edge less than two quadrants.

\section{Statistical Analysis}

Statistical analysis was performed using StataSE15 (version 15.0, Stata Corp LP, TX, USA). All data were presented as means (standard deviations) or counts and percentages according to data type. Trends in corrected distance visual acuity (CDVA) of different PCO grades were assessed using the Wilcoxon-type non-parametric test for trend across different PCO groups. Differences between with PCO group and without PCO group were compared by independent $t$ test and Pearson chi-square test. Univariate and multivariate logistic regression analyses were performed to determine the risk factors of PCO. A $P$ value less than 0.05 was considered to be statistically significant.

\section{RESULTS}

A total of 1039 eyes of 1039 participants (mean [SD] age, 66.68 [11.43] years; 437 [42.06\%] male) were enrolled in this study. The main diagnosis (74.98\%) was age-related cataract, and $25.02 \%$ were complicated cataract. There were 798 patients who had hydrophobic acrylic IOL implanted, of which $83.96 \%$ were square-edge IOL. Of 241 patients with implanted hydrophilic acrylic IOL, 229 patients (95.02\%) chose square-edge IOL with $360^{\circ}$ posterior barrier. Table 1 shows the basic characteristics of the patients.

As illustrated in Table 2, the prevalence of early-onset PCO was 29.93\% (311/1039) in all the patients at 3 months after cataract surgery, while the prevalence was higher in patients with complicated cataracts than that in patients with age-related cataracts $(40.38 \%$ [105/260] vs $26.44 \%$ [206/779]). There were statistically significant differences in the distribution of different levels of PCO between age-related cataract and complicated cataract $(P<0.001)$. In particular, the incidence of grade $3+\mathrm{PCO}$ in complicated cataract was obviously higher than that in age-related cataract $(8.08 \%$ [21/260] vs $1.28 \%[10 / 779])$.

In order to assess visual impact caused by PCO, participants with previous ocular diseases or surgery that might affect visual acuity, such as high myopia ( $\mathrm{AL} \geq 26 \mathrm{~mm}$ ), fundus diseases, glaucoma, and previous PPV surgery, were excluded from the analysis. There were 147 eyes from 147 patients with age-related cataracts with PCO eligible for this analysis. As shown in Table 3, corrected distance visual acuity (CDVA) declined with the severity of PCO $(P$ trend $=0.011)$, and PCO mainly induced slight visual impairment $(P$ trend $=0.015)$.

Table 4 compares the characteristics of patients with or without PCO. Patients with PCO were younger than those without PCO (mean [SD], 64.79 [12.20] years vs 67.50 [11.01], $P=0.001)$. Additionally, the incidence of PCO 
Table 1 Characteristics of participants

\begin{tabular}{|c|c|}
\hline Characteristics & Values \\
\hline Patients, $n$ & 1039 \\
\hline Age (years), mean (SD) & $\begin{array}{l}66.68 \\
\quad(11.43)\end{array}$ \\
\hline Male, $n(\%)$ & $437(42.06)$ \\
\hline Smoking, $n(\%)$ & $97(9.34)$ \\
\hline Drinking, $n(\%)$ & $125(12.03)$ \\
\hline BMI, mean (SD) & $23.79(3.53)$ \\
\hline Diabetes, $n(\%)$ & $170(16.36)$ \\
\hline Hypertension, $n(\%)$ & $349(33.59)$ \\
\hline Right eyes & $663(63.81)$ \\
\hline Previous PPV surgery, $n(\%)$ & $54(5.20)$ \\
\hline Anterior chamber depth (mm), mean (SD) & $3.16(0.47)$ \\
\hline Axial length (mm), mean (SD) & $24.33(2.30)$ \\
\hline Axial length $\geq 26.0 \mathrm{~mm}, n(\%)$ & $161(15.50)$ \\
\hline \multicolumn{2}{|l|}{ Cataract types } \\
\hline Age-related cataract, $n(\%)$ & $779(74.98)$ \\
\hline Complicated cataract, $n(\%)$ & $260(25.02)$ \\
\hline \multicolumn{2}{|l|}{ IOL types } \\
\hline Hydrophilic IOL, $n$ (\%) & $241(23.20)$ \\
\hline Hydrophobic IOL, $n$ (\%) & $798(76.80)$ \\
\hline \multicolumn{2}{|c|}{ Classification of capsulorhexis overlap IOL edges } \\
\hline $360^{\circ}$ overlap, $n(\%)$ & $419(40.33)$ \\
\hline $180-360^{\circ}$ overlap, $n(\%)$ & $488(46.97)$ \\
\hline$<180^{\circ}$ overlap, $n(\%)$ & $132(12.70)$ \\
\hline
\end{tabular}

was much higher in patients with PPV surgery history $(53.70 \%$ [29/54] vs $28.63 \%$ [282/985], $P<0.001)$ and incomplete capsulorhexis-IOL overlap $\left(<180^{\circ}\right.$ overlap: $56.06 \%$ [74/132]; 180-360 ${ }^{\circ}$ overlap: $32.17 \% \quad[157 / 488] ; \quad 360^{\circ}$ overlap: $19.09 \%$ [80/419]; $P<0.001)$. Moreover, patients with $\mathrm{PCO}$ had longer AL than those without PCO (mean [SD], 24.56 [2.48] $\mathrm{mm}$ vs
$24.24[2.22] \mathrm{mm}, P=0.042)$. However, the incidence of PCO showed no statistically significant differences in gender $(P=0.804)$, smoking $(P=0.818)$, drinking $(P=0.419)$, BMI $(P=0.863)$, hypertension $(P=0.225)$, diabetes $(P=0.208)$, and IOL types $(P=0.074)$.

The results of univariate and multivariate logistic regression analysis are presented in Table 5. The univariate logistic regression analysis demonstrated that previous PPV surgery (OR 2.892, 95\% CI 1.664-5.025; $P<0.001$ ) and the deficiency of capsulorhexis-IOL overlap were risk factors of PCO $\left(180-360^{\circ}\right.$ overlap: OR 2.010 , 95\% CI 1.476-2.737, $P<0.001 ;<180^{\circ}$ overlap: OR 5.406, 95\% CI 3.548-8.240, $P<0.001)$. However, age was a protective factor of PCO (OR 0.980, 95\% CI 0.969-0.991; $P=0.001)$.

Multivariate logistic regression analysis showed that previous PPV surgery and incomplete capsulorhexis-IOL overlap were positively associated with PCO (PPV: OR 2.664, 95\% CI 1.409-5.037, $P=0.003 ; 180-360^{\circ}$ overlap: OR 2.058, 95\% CI 1.486-2.850, $P<0.001 ;<180^{\circ}$ overlap: OR 5.403, 95\% CI 3.436-8.496, $P<0.001)$. Although hydrophilic IOL was negatively associated with PCO (OR $0.610,95 \%$ CI $0.427-0.875 ; P=0.007$ ), the rates of PCO were not significantly different between hydrophilic IOL and hydrophobic IOL groups $(P=0.074$, Table 4). Nevertheless, age, gender, smoking, drinking, BMI, hypertension, and diabetes showed no association with PCO. Taken together, these results suggested that previous PPV surgery and lager capsulorhexis with insufficient IOL overlap are the significant risk factors for early-onset PCO.

\section{DISCUSSION}

Even though several studies focusing on the effects of specific factors on the incidence of long-term PCO have been performed, such as capsulotomy sizes and IOL types, there was scarce evidence on how multiple factors affect incidence of early-onset PCO. In this study, we found that PCO could occur a short time after cataract surgery. Approximately 2.98\% of patients had grade $3+$ PCO 3 months after 
Table 2 Distribution of early-onset posterior capsule opacification in age-related cataract and complicated cataract

\begin{tabular}{lllll}
\hline PCO grades & Total, $(\boldsymbol{n}, \boldsymbol{\%})$ & Age-related cataract, $\boldsymbol{n}(\%)$ & Complicated cataract, $\boldsymbol{n}(\%)$ & $\boldsymbol{P}$ value \\
\hline Without PCO & $728(70.07)$ & $573(73.56)$ & $155(59.62)$ \\
With PCO & $311(29.93)$ & $206(26.44)$ & $105(40.38)$ \\
Grade 1 & $208(20.02)$ & $157(20.15)$ & $51(19.61)$ \\
Grade 2 & $72(6.93)$ & $39(5.01)$ & $33(12.69)$ \\
Grade 3 + grade 4 & $31(2.98)$ & $10(1.28)$ & $21(8.08)$ \\
Total & 1039 & 779 & 260
\end{tabular}

$<0.001$

PCO posterior capsule opacification

Table 3 Influences of posterior capsule opacification on visual impairment in age-related cataract

\begin{tabular}{lcccc}
\hline CDVA (logMAR) & Grade 1 & Grade 2 & Grade 3 + grade 4 & P trend \\
\hline Total, mean (SD) & $0.12(0.21)$ & $0.18(0.18)$ & $0.3(0.41)$ & 0.011 \\
CDVA $\leq 0.1(20 / 25), n(\%)$ & $90(78.95)$ & $16(59.26)$ & $3(50.00)$ & 0.537 \\
$0.1(20 / 25)<$ CDVA $\leq 0.3(20 / 40), n(\%)$ & $16(14.04)$ & $6(22.22)$ & $2(33.33)$ & 0.015 \\
CDVA $>0.3(20 / 40), n(\%)$ & $8(7.01)$ & $5(18.52)$ & $1(16.67)$ & 0.957 \\
\hline
\end{tabular}

$P C O$ posterior capsule opacification, $C D V A$ corrected distance visual acuity

surgery, and the incidence was even higher in patients with complicated cataract $(8.08 \%)$. Moreover, we also determined that previous PPV surgery and incomplete capsulorhexis-IOL overlap were risk factors for early-onset PCO. Patients with previous PPV surgery and insufficient capsulorhexis-IOL overlap had much higher rates of PCO.

The incidence of early-onset PCO has been reported to range from $11 \%$ to $40.7 \%$ in patients with cataracts with silicone IOL implantation at 6 weeks to 3 months after cataract surgery in previous studies $[17,18]$. However, no study reported the incidence of short-term PCO for acrylic acid IOL implantation. In our study, 29.93\% of patients developed early-onset PCO 3 months after cataract surgery, of which $66.88 \%$ with PCO presented grade 1 that only involved the peripheral posterior capsule and did not affect visual acuity. The incidence of grade $2+$ PCO in our study was $9.91 \%$, including $2.98 \%$ of grade 3 and grade 4 PCO affecting vision. These results implied that the incidence of early-onset PCO should not be ignored.

It is known that age is a lingering factor associated with PCO. The pathogenesis of PCO is caused by the residual LEC proliferation, migration, and differentiation after cataract surgery [19]. Sundelin et al. reported that the process of PCO formation was more active in younger patients in a vitro study [20]. Tokko and colleagues also demonstrated that age was negatively associated with the need for YAG capsulotomy [13]. Though there was no significant correlation between age and PCO in multivariate analysis in our study, patients with PCO were significantly younger than those without PCO. Therefore, age may be associated with PCO, particularly for long-term PCO, but is not a major risk factor for early-onset PCO. 
Table 4 Characteristics of patients with and without posterior capsule opacification

\begin{tabular}{lllll}
\hline & Total & Without PCO & With PCO & P value \\
\hline Patients, $n$ (\%) & 1039 & $728(70.07)$ & $311(29.93)$ & - \\
Age (years), mean (SD) & 1039 & $67.50(11.01)$ & $64.79(12.20)$ & 0.001 \\
BMI, mean (SD) & 1039 & $23.78(3.67)$ & $23.82(3.17)$ & 0.863 \\
Axial length (mm), mean (SD) & 1039 & $24.24(2.22)$ & $24.56(2.48)$ & 0.042 \\
Gender, $n(\%)$ & & & & \\
Male & 437 & $308(70.48)$ & $129(29.52)$ & \\
Female & 602 & $420(69.77)$ & $182(30.23)$ &
\end{tabular}

Smoking, $n(\%)$

$\begin{array}{llll}\text { Yes } & 97 & 69(71.13) & 28(28.87) \\ \text { No } & 942 & 659(69.69) & 283(30.04)\end{array}$

Drinking, $n(\%)$

$\begin{array}{llll}\text { Yes } & 125 & 91(72.80) & 34(27.20) \\ \text { No } & 914 & 637(69.69) & 277(30.31)\end{array}$

Hypertension (yes)

Yes

No

349

690

$253(72.49)$

$475(68.84)$

$96(27.51)$

$215(31.16)$

0.225

Diabetes (yes)

Yes

No

170

$126(74.12)$

44 (25.88)

869

602 (69.28)

267 (30.72)

0.208

Previous PPV surgery, $n$ (\%)

Yes

No

54

985

25 (46.30)

$29(53.70)$

$282(28.63)$

$<0.001$

IOL types

Hydrophilic, $n$ (\%)

241

Hydrophobic, $n$ (\%)

798

180 (74.69)

$61(25.31)$

$250(31.33)$

Classification of capsulorhexis overlap IOL edges 
Table 4 continued

\begin{tabular}{lllll}
\hline & Total & Without PCO & With PCO & P value \\
\hline $360^{\circ}$ overlap & 419 & $339(80.91)$ & $80(19.09)$ & \\
$180-360^{\circ}$ overlap & 488 & $331(67.83)$ & $157(32.17)$ & \\
$<180^{\circ}$ overlap & 132 & $58(43.94)$ & $74(56.06)$
\end{tabular}

$<0.001$

$P C O$ posterior capsule opacification, $B M I$ body mass index, $P P V$ pars plana vitrectomy, $I O L$ intraocular lens

Table 5 Univariate and multiple logistic regression analysis of risk factors associated with early-onset posterior capsule opacification

\begin{tabular}{|c|c|c|c|c|}
\hline \multirow[t]{3}{*}{ Factors } & \multicolumn{4}{|l|}{ PCO } \\
\hline & \multicolumn{2}{|l|}{ Univariate analysis } & \multicolumn{2}{|l|}{ Multivariate analysis } \\
\hline & Odds ratio $(95 \% \mathrm{Cl})$ & $P$ & Odds ratio $(95 \% \mathrm{Cl})$ & $P$ \\
\hline Age (years) & $0.980(0.969-0.991)$ & 0.001 & $0.987(0.973-1.000)$ & 0.058 \\
\hline Gender (male) & $1.035(0.790-1.354)$ & 0.804 & $1.022(0.738-1.416)$ & 0.896 \\
\hline Smoking (yes) & $1.052(0.683-1.620)$ & 0.818 & $1.037(0.627-1.715)$ & 0.887 \\
\hline Drinking (yes) & $0.841(0.553-1.280)$ & 0.419 & $0.704(0.434-1.141)$ & 0.155 \\
\hline BMI & $1.003(0.966-1.043)$ & 0.863 & $1.000(0.957-1.044)$ & 0.982 \\
\hline Hypertension (yes) & $0.838(0.631-1.115)$ & 0.225 & $0.883(0.629-1.240)$ & 0.473 \\
\hline Diabetes (yes) & $0.787(0.543-1.142)$ & 0.208 & $0.865(0.573-1.307)$ & 0.492 \\
\hline Previous PPV surgery (yes) & $2.892(1.664-5.025)$ & $<0.001$ & $2.664(1.409-5.037)$ & 0.003 \\
\hline Axial length (mm) & $1.059(1.002-1.120)$ & 0.042 & $1.056(0.993-1.122)$ & 0.081 \\
\hline IOL types (hydrophilic) & $0.743(0.536-1.030)$ & 0.074 & $0.610(0.427-0.875)$ & 0.007 \\
\hline \multicolumn{5}{|c|}{ Classification of capsulorhexis-IOL overlap } \\
\hline $360^{\circ}$ overlap & Ref. & & Ref. & \\
\hline $180-360^{\circ}$ overlap & $2.010(1.476-2.737)$ & $<0.001$ & $2.058(1.486-2.850)$ & $<0.001$ \\
\hline$<180^{\circ}$ overlap & $5.406(3.548-8.240)$ & $<0.001$ & $5.403(3.436-8.496)$ & $<0.001$ \\
\hline
\end{tabular}

$P C O$ posterior capsule opacification, $B M I$ body mass index, $P P V$ pars plana vitrectomy, $I O L$ intraocular lens

Another important finding in our study was that previous PPV surgery was a significant risk factor for early-onset PCO. Previous studies also reported that the incidence of PCO was $33.3-50 \%$ in vitrectomized eyes at about
2-3 years after cataract surgery [21, 22]. Pinter and Sugar further pointed out that PCO was more common in vitrectomized eyes than in control eyes (51\% vs 21\%) for about 2 years follow-up [21]. In our study, 29 of 54 patients 
(53.70\%) with a history of PPV surgery had grade $1+$ PCO only 3 months after cataract surgery, including 9 patients (16.67\%) with grade $3+$ PCO affecting vision. A possible explanation for the increasing risk of PCO in vitrectomized eyes is that elevated cytokines caused by inflammation after PPV surgery may accelerate LEC proliferation and migration, which results in PCO development. Additionally, loss of support of the vitreous body may affect the attachment between IOL edge and capsular bag, promoting rapid LEC proliferation and contributing to PCO [23]. As a result of the high incidence of PCO in patients with previous PPV surgery, previous study has proposed that PCCC combined with phacoemulsification may be an alternative to prevent PCO in postvitrectomy eyes with cataract [24].

Previous studies have found capsulorhexis size and its central localization play important roles in PCO $[25,26]$. Small capsulorhexis $(4.0-5.0 \mathrm{~mm})$ that covers the IOL edge perfectly had a lower PCO rate than large capsulorhexis $[25,26]$. In this study, we also found that larger capsulorhexis with incomplete capsulorhexis-IOL overlap was a significant risk factor for early-onset PCO. The complete in-thebag fixation of IOL provides accurate lens centration and enhances the IOL-optic barrier effect; on the contrary, incomplete capsulorhexis-IOL overlap may provide potential space for the migration and growth of LECs onto the posterior capsule and contributes to PCO formation. Hence, a centered and appropriately sized capsulorhexis that covers the IOL edge completely is critical for cataract surgery.

In the current study, we found that the incidence of PCO was slightly lower in hydrophilic IOL than that in hydrophobic IOL, and hydrophilic IOL was negatively associated with PCO in multivariate analysis. These findings seem to contradict previous research reporting low PCO rates in patients with hydrophobic IOL implantation $[27,28]$. The main reason may be that $95.02 \%$ of hydrophilic IOLs used in this study were $360^{\circ}$ enhanced sharp-edged hydrophilic IOLs. Koshy et al. ascertained that $360^{\circ}$ enhanced sharp-edged hydrophilic IOL had a better capsular bag performance, and a lower PCO incidence than sharp-edged hydrophobic
IOL in the 2 years after cataract surgery though PCO scoring showed no statistically significant difference between the two groups [29]. These results suggested that the design of IOL may be more important than the material in reducing the incidence of PCO.

This study has several limitations. Since this is a cross-sectional study, the dynamic changes of early-onset PCO and how long grade 1 PCO will need Nd:YAG capsulotomy therapy are unknown. Therefore, a well-designed longitudinal study is necessary to further explore the progression and the associated factors of PCO.

\section{CONCLUSIONS}

In the present cross-sectional study, we propose that PCO can occur shortly following uneventful cataract surgery, thus affecting the postoperative visual quality and reducing patient satisfaction. Approximately $1.28 \%$ of age-related cataract and $8.08 \%$ of patients with complicated cataracts with grade $3+$ PCO required Nd:YAG capsulotomy therapy only 3 months after surgery. And CDVA declined with the increase in PCO grades. Moreover, we also demonstrated that previous PPV surgery and incomplete capsulorhexis-IOL overlap were the significant risk factors for early-onset PCO. Therefore, a centered and appropriately sized capsulorhexis that covers the IOL edge perfectly is critical for cataract surgery, especially for patients with previous PPV surgery. Furthermore, for patients with PPV surgery history, prophylactic PCCC can be performed during cataract surgery, so as to avoid the need for Nd:YAG capsulotomy therapy early after surgery and reduce its complications.

\section{ACKNOWLEDGEMENTS}

We thank the participants of the study.

Funding. Sponsorship for this study and the journal's Rapid Service Fee were funded by the National Natural Science Foundation of China $(81770905,81873675,81700820)$, the Pearl 
River Nova Program of Guangzhou (201806010167), and the Construction Project of High-Level Hospitals in Guangdong Province (303020102).

Authorship. All named authors meet the International Committee of Medical Journal Editors (ICMJE) criteria for authorship for this article, take responsibility for the integrity of the work as a whole, and have given their approval for this version to be published.

Author Contributions. Xiaoxun $\mathrm{Gu}$ and Xiaoyun Chen are both first authors, who planned the study, collected the data, analyzed the data and prepared this paper. Guangming Jin and Lanhua Wang planned the study, analyzed the data. Enen Zhang collected the data. Wei Wang reviewed and contributed to the content of this paper. Zhenzhen Liu planned the study, reviewed and contributed to the content of this paper. Lixia Luo planned the study, reviewed and contributed to the content of this paper.

Disclosures. Xiaoxun $\mathrm{Gu}$, Xiaoyun Chen, Guangming Jin, Lanhua Wang, Enen Zhang, Wei Wang, Zhenzhen Liu, Lixia Luo declare that they have no conflicts of interest.

Compliance with Ethics Guidelines. This cross-sectional study was conducted at Zhongshan Ophthalmic Center, Sun Yat-sen University, Guangzhou, China. It was approved by the Zhongshan Ophthalmic Center Institutional Review Board (No. 2019 KYPJ033) and was conducted in accordance with the Declaration of Helsinki. Written informed consent was obtained from each participant.

Data Availability. The datasets used and/or analyzed during the current study are available from the corresponding author upon reasonable request.

Open Access. This article is licensed under a Creative Commons Attribution-NonCommercial 4.0 International License, which permits any non-commercial use, sharing, adaptation, distribution and reproduction in any medium or format, as long as you give appropriate credit to the original author(s) and the source, provide a link to the Creative Commons licence, and indicate if changes were made. The images or other third party material in this article are included in the article's Creative Commons licence, unless indicated otherwise in a credit line to the material. If material is not included in the article's Creative Commons licence and your intended use is not permitted by statutory regulation or exceeds the permitted use, you will need to obtain permission directly from the copyright holder. To view a copy of this licence, visit http://creativecommons.org/licenses/by$\mathrm{nc} / 4.0 /$.

\section{REFERENCES}

1. Ursell PG, Dhariwal M, O'Boyle D, Khan J, Venerus A. 5 year incidence of YAG capsulotomy and PCO after cataract surgery with single-piece monofocal intraocular lenses: a real-world evidence study of 20,763 eyes. Eye (Lond). 2020;34(5):960-8.

2. Wormstone IM, Wormstone YM, Smith AJO, Eldred JA. Posterior capsule opacification: what's in the bag? Prog Retin Eye Res. 2020;82:100905.

3. Lu B, Zhu W, Fan Y, Shi D, Ma L. Utility of the optical quality analysis system for decision-making in Nd:YAG laser posterior capsulotomy in patients with light posterior capsule opacity. BMC Ophthalmol. 2021;21(1):7.

4. Ari S, Cingu AK, Sahin A, Cinar Y, Caca I. The effects of Nd:YAG laser posterior capsulotomy on macular thickness, intraocular pressure, and visual acuity. Ophthal Surg Lasers Imaging. 2012;43(5): 395-400.

5. Leydolt C, Schriefl S, Stifter E, Haszcz A, Menapace R. Posterior capsule opacification with the iMics1 NY-60 and AcrySof SN60WF 1-piece hydrophobic acrylic intraocular lenses: 3-year results of a randomized trial. Am J Ophthalmol. 2013;156(2): 375-81.

6. Wesolosky JD, Tennant M, Rudnisky CJ. Rate of retinal tear and detachment after neodymium:YAG capsulotomy. J Cataract Refract Surg. 2017;43(7): 923-8.

7. Dhariwal M, Bouchet C, Jawla S. Comparing the long-term impact on health care resources utilization and costs due to various single-piece acrylic 
monofocal intraocular lens implantation during cataract surgery: a cost-consequence analysis for the United Kingdom, Italy, and Denmark. Clin Ophthalmol. 2019;13:169-76.

8. Raulinajtys-Grzybek M, Grabska-Liberek I, Opala A, Slomka M, Chrobot M. Budget impact analysis of lens material on the posterior capsule opacification (PCO) as a complication after the cataract surgery. Cost Effect Resour Alloc. 2020;18:19. https://doi. org/10.1186/s12962-020-00214-y.

9. Vock L, Menapace R, Stifter E, Buhl W, Georgopoulos M. Effect of primary posterior continuous curvilinear capsulorhexis on clinical performance of ACR6D SE single-piece hydrophilic acrylic intraocular lenses. J Cataract Refract Surg. 2007;33(4):628-34.

10. Al-Nashar HY, Khalil AS. Primary posterior capsulotomy in adults with posterior capsule opacification. J Cataract Refract Surg. 2016;42(11):1615-9.

11. De Groot V, Hubert M, Van Best JA, Engelen S, Van Aelst S, Tassignon MJ. Lack of fluorophotometric evidence of aqueous-vitreous barrier disruption after posterior capsulorhexis. J Cataract Refract Surg. 2003;29(12):2330-8.

12. Monestam E. Long-term outcome of cataract surgery: 20-year results from a population-based prospective study. J Cataract Refract Surg. 2019;45(12):1732-7.

13. Tokko HA, Hussain F, Al-Awadi A, et al. Factors associated with the development of posterior capsule opacification requiring yttrium aluminum garnet capsulotomy. Optom Vision Sci. 2019;96(7):492-9.

14. Liu Z, Cao Q, Qu B, et al. Fluid-jet technique to polish the posterior capsule for phacoemulsification surgeries: efficacy and safety evaluation. J Cataract Refract Surg. 2020;46(11):1508-14.

15. Chen X, Gu X, Wang W, et al. Characteristics and factors associated with intraocular lens tilt and decentration after cataract surgery. J Cataract Refract Surg. 2020;46(8):1126-31.

16. Congdon N, Fan H, Choi K, et al. Impact of posterior subcapsular opacification on vision and visual function among subjects undergoing cataract surgery in rural China: Study of Cataract Outcomes and Up-Take of Services (SCOUTS) in the Caring is Hip Project, Report 5. Brit J Ophthalmol. 2008;92(5):598-603.

17. Mootha VV, Tesser R, Qualls C. Incidence of and risk factors for residual posterior capsule opacification after cataract surgery. J Cataract Refract Surg. 2004;30(11):2354-8.

18. Alio JL, Plaza-Puche AB, Montalban R, Ortega P. Near visual outcomes with single-optic and dual- optic accommodating intraocular lenses. J Cataract Refract Surg. 2012;38(9):1568-75.

19. Koch CR, D'Antin JC, Tresserra F, Barraquer RI, Michael R. Histological comparison of in vitro and in vivo development of peripheral posterior capsule opacification in human donor tissue. Exp Eye Res. 2019;188:107807.

20. Sundelin K, Petersen A, Soltanpour Y, Zetterberg M. In vitro growth of lens epithelial cells from cataract patients-association with possible risk factors for posterior capsule opacification. Open Ophthalmol J. 2014;8:19-23.

21. Pinter SM, Sugar A. Phacoemulsification in eyes with past pars plana vitrectomy: case-control study. J Cataract Refract Surg. 1999;25(4):556-61.

22. Hocaoglu M, Karacorlu M, Muslubas IS, Ozdemir H, Arf S, Uysal O. Incidence and factors associated with complications of sutured and sutureless cataract surgery following pars plana vitrectomy at a tertiary referral centre in Turkey. Brit J Ophthalmol. 2016;100(9):1206-10.

23. Jun JH, Kim KS, Chang SD. Nd:YAG capsulotomy after phacoemulsification in vitrectomized eyes: effects of pars plana vitrectomy on posterior capsule opacification. J Ophthalmol. 2014;2014:840958.

24. Yu MT, Yan D, Wu WJ, Wang YB, Wu XN. Clinical outcomes of primary posterior continuous curvilinear capsulorhexis in postvitrectomy cataract eyes. J Ophthalmol. 2020;2020:1-7.

25. Aykan U, Bilge AH, Karadayi K, Akin T. The effect of capsulorhexis size on development of posterior capsule opacification: small $(4.5-5.0 \mathrm{~mm})$ versus large (6.0-7.0 mm). Eur J Ophthalmol. 2003;13(6): 541-5.

26. Langwinska-Wosko E, Broniek-Kowalik K, Szulborski K. The impact of capsulorhexis diameter, localization and shape on posterior capsule opacification. Med Sci Monit. 2011;17(10):Cr577-82.

27. Li Y, Wang J, Chen Z, Tang X. Effect of hydrophobic acrylic versus hydrophilic acrylic intraocular lens on posterior capsule opacification: meta-analysis. PLoS ONE. 2013;8(11):e77864.

28. Zhao Y, Yang K, Li J, Huang Y, Zhu S. Comparison of hydrophobic and hydrophilic intraocular lens in preventing posterior capsule opacification after cataract surgery: an updated meta-analysis. Medicine (Baltimore). 2017;96(44):e8301.

29. Koshy J, Hirnschall N, Vyas AKV, et al. Comparing capsular bag performance of a hydrophilic and a hydrophobic intraocular lens: a randomised twocentre study. Eur J Ophthalmol. 2018;28(6):639-44. 